\title{
Comparison of the Upper and Lower Trapezius and Serratus Anterior during Push-up Plus Exercise with and without an Elastic Shoulder Band
}

\author{
Kyeong-Jin Kim $\cdot$ Hye-Jin Woo $\cdot$ Yu-Jeong Hong $\cdot$ Young-In Hwang ${ }^{\dagger}$
}

Department of Physical Therapy, College of Life and Health Science, Hoseo University

Received: February 28, 2018 / Revised: March 8, 2018 / Accepted: April 6, 2018

(C) 2018 J Korean Soc Phys Med

\section{| Abstract |}

PURPOSE: Scapular stability is very important to arm function during activities of daily living. The push-up plus exercise is commonly used to stabilize the shoulder muscles. In the present study, we designed an elastic shoulder band (ESB) that could be used with the push-up plus exercise and studied muscle activities of the serratus anterior (SA) and the lower and the upper trapezius (LT and UT) on stable and unstable support.

METHODS: Fifteen healthy people participated in this study. Three muscles were investigated using surface EMG with and without the ESB on stable and unstable support. All subjects performed each trial three times for 7 seconds per set and rested for 30 seconds. Repeated one-way ANOVA was used for statistical analyses.

RESULTS: The EMG activity of the SA was significantly different during the push-up plus exercise on stable support with the ESB compared with that on unstable support without

$\dagger$ Corresponding Author : Young-In Hwang young123@hoseo.edu, http://orcid.org/0000-0002-7314-1678 This is an Open Access article distributed under the terms of the Creative Commons Attribution Non-Commercial License (http://creativecommons.org/licenses/by-nc/3.0) which permits unrestricted non-commercial use, distribution, and reproduction in any medium, provided the original work is properly cited. the $\operatorname{ESB}(p<.05)$. However, there were no differences in the activities of UT and LT on stable and unstable support ( $p>.05)$. CONCLUSION: The ESB was effective for the activity of SA during the push-up plus exercise on stable support; therefore, it has the potential for use by people during push-up plus exercises. However, further studies are required to investigate the rehabilitation tools for patients with shoulder instability.

Key Words: EMG, Muscle activity, Scapula

\section{Introduction}

It is important for the scapula to be stable to ensure shoulder stability and upper extremity function (Cools et al., 2007a). The scapula is closely related to movements of the upper extremity, especially overhead activity (Kibler, 1998; Burkhart et al., 2003). For the scapula to function well, the muscles that support it are essential as they provide proximal stability and fixation during the movement of proximal parts, such as the arms (Voight and Thomson, 2000). The scapula is fixed within the thorax, and scapulothoracic (ST) movement occurs with movements of the shoulder. If the appropriate ST movement does not occur, this risk of pathologic movement increases, and efforts are made to restore normal, stable movement 
through movement of the gleno-humeral joint itself (Bigliani et al., 1997; Kamkar et al., 1993).

To prevent pathological movement of the scapula, it is important to prevent deterioration of neurologic control and strength in the muscles that move and support the scapula. In this region, SA, UT, and LT produce the force required for coupled motion and are able to regulate the scapula with upward and posterior tilt (Hsu et al., 2009; Cools et al., 2007b). Impaired SA muscle function is usually present in individuals with shoulder pathology (Ludewig and Cook, 2000); therefore, there is a need for exercises that increase SA activity.

Exercises related to scapular stabilization include lawnmowers, the robber exercise, the bow and arrow exercise, the external-rotation-with-scapular-squeeze exercise, and the push-up plus exercise (Moller et al., 2014). These exercises are often performed with an elastic band to provide variable resistance without interfering with movement. For example, tying is used to support the neck (Yoo and Yoo, 2013) similar to the exercises with a low row thera-band, the dynamic hug exercise, and scapular punches (Paine and Voight, 2013). The push-up plus exercise is most commonly used to stabilize the scapula because it is better than other exercises at improving SA activity (Kim et al., 2014). However, there are different opinions regarding whether the push-up plus exercise should be conducted on stable or unstable support. Lee et al. (2013) reported that performing the exercise on unstable support was more effective at increasing muscle activity and stimulating shoulder joint proprioceptors. However, Lehman et al. (2008) claimed that performing the exercise on unstable support had little effect.

Thus, in the present study, we redesigned an existing elastic band as a 'shoulder elastic band' that could be used in shoulder stabilization exercises. We then applied this band to the push-up plus exercise and investigated its effect on the activity of scapula stabilizing muscles when performing the exercise on stable or unstable support.

\section{Methods}

\section{Subjects}

In this study, we measured the muscle activity of the shoulder stabilizing muscles in 15 healthy adults when performing the push-up plus exercise on stable or unstable support, while wearing or not wearing the ESB. Before beginning the study, all subjects were informed about the full procedure and all gave their voluntary, written consent. There were 15 subjects in total (nine males and six females). The mean age was $22.86 \pm .83$ years, the mean height was $173.7 \pm 6.3 \mathrm{~cm}$, and the mean body weight was $67.6 \pm 16.4$ $\mathrm{kg}$. All subjects were physically healthy adults, aged 20-29 years (Table 1). Exclusion criteria were individuals with a congenital deformity causing elevated scapulae or inability to depress the scapulae (Sprengel's deformity), scapular winging, contra-indication for EMG, or laceration.

\section{Measurement}

We used surface EMG (Trigno Wireless EMG system, Delsys Inc., USA) to examine muscle activity, focusing on the three upper extremity muscles (UT, LT, and SA). The EMG electrode attachment points for these three muscles were as follows: for the UT, slightly lateral to the point halfway between the $7^{\text {th }}$ cervical vertebra and the acromion; for the LT, approximately $5 \mathrm{~cm}$ inferior to the scapular spine; for the SA, at the level of the inferior tip of the scapula, approximately at the mid-axillary line over the $5^{\text {th }}$ rib on the right side (Seo et al., 2013) (Fig. 1).

To normalize the muscle activities of the UT, LT, and SA across different exercise methods, we analyzed muscle activity as the percentage of maximal voluntary isometric Table 1. General Characteristics of Subjects

\begin{tabular}{cc}
\hline & Mean \pm SD \\
\hline Age (years) & $22.86 \pm .83$ \\
Weight $(\mathrm{kg})$ & $67.60 \pm 16.40$ \\
Height $(\mathrm{cm})$ & $173.70 \pm 6.30$ \\
\hline
\end{tabular}




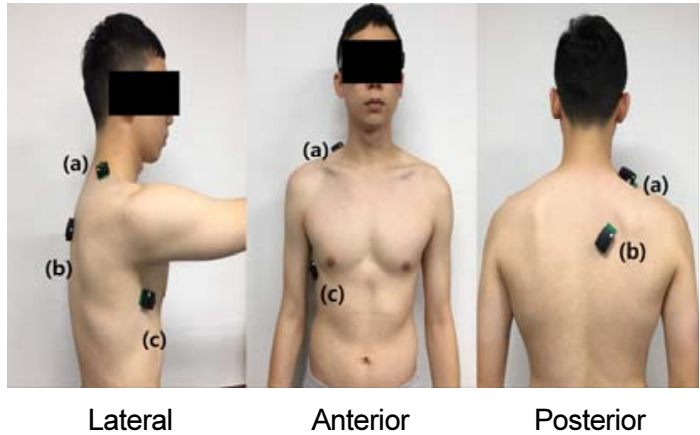

Fig. 1. EMG attachment : (a) upper trapezius, (b) lower trapezius, (c) serratus anterior

contraction (\%MVIC), which was calculated by measuring the root mean square (RMS), and compared it to the activity measured at maximal voluntary isometric contraction (MVIC). To determine the location of the MVIC, we used the method described by McLean and Kendal. For the UT, the measurement was taken from the center of the head with the shoulders spread at $90^{\circ}$. For the LT, the measurement was taken with the shoulders rotated externally and the arms spread at $125^{\circ}$ while applying resistance far from the elbow. For SA, the measurement was taken with the subject sitting on a table, the shoulders rotated internally, and the scapular surfaces spread at $125^{\circ}$ while applying resistance close to the elbow.

\section{Procedure}

For the ESB, we used a silver-level band, measured a length equal to the distance between the scapulae (average $10-15 \mathrm{~cm}$ ), cut the remaining length in half, and tied knots at either end. The knots were placed underneath the axillae, and another knot was tied halfway between the scapular spines to improve shoulder stability (Fig. 2).

For the push-up plus position, the subjects first adopted a comfortable posture with their arms spread at shoulder-width. Next, paper was attached to each middle finger to fix them in place. Placing their knees on the ground leaving a gap of $10 \mathrm{~cm}$ between them, the subjects bent their hips and knees at $90^{\circ}$. A smartphone application

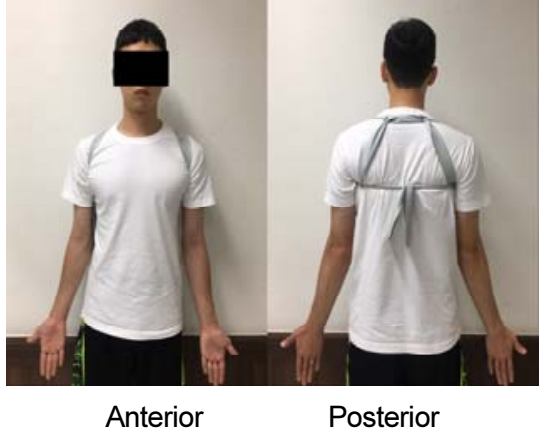

Fig. 2. Elastic shoulder band

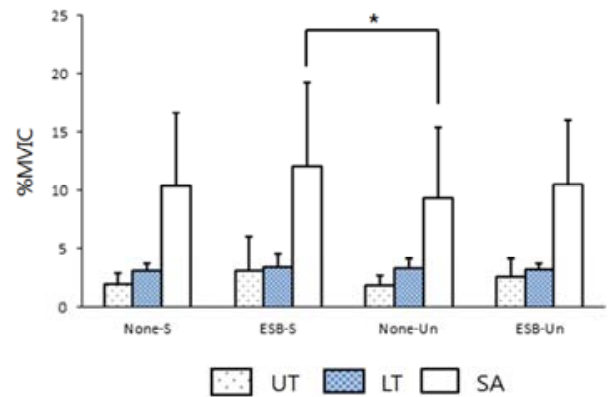

Fig. 3. Post-hoc tests of UT, LT and SA on stable and unstable support with and without ESB

called 'Inclinometer Free' was used to ensure that the shoulder and elbow joints were vertical, and to make sure that the difference in the slopes was no more than $5^{\circ}$. When the investigator gave the 'start' command, the subject protracted their scapulae for 7 seconds by trying to roll their shoulders forward, after which they retracted their scapulae. Before performing the above experiment, the three previously investigated muscles were stretched. The order of each trial was randomly selected by each subject.

Subjects conducted each set for 7 seconds, then rested for 30 seconds. Three sets were performed without the ESB and three sets with the ESB on the stable and unstable surface. The mean muscle activity was compared during the 5 seconds of contraction excluding the first and last second. In this study, we partially applied the push-up plus exercise, involving scapular retraction, followed by maintenance of isometric contraction. This allows the 
Table 2. Muscle Activities on Stable and Unstable Support with and without ESB

\begin{tabular}{|c|c|c|c|c|c|}
\hline & Sta & port & Uns & support & \multirow{2}{*}{$\mathrm{P}$} \\
\hline & None-S & ESB-S & None-Un & ESB-Un & \\
\hline UT & $1.95 \pm .91$ & $3.14 \pm 2.91$ & $1.86 \pm .76$ & $2.56 \pm 1.55$ & .30 \\
\hline LT & $3.12 \pm .56$ & $3.39 \pm 1.13$ & $3.32 \pm .84$ & $3.18 \pm .51$ & .66 \\
\hline SA & $10.43 \pm 6.19$ & $12.02 \pm 7.27$ & $9.33 \pm 6.10$ & $10.45 \pm 5.56$ & $.03 *$ \\
\hline
\end{tabular}

None-S: no elastic shoulder band with stable support, ESB-S: elastic shoulder band with stable support, None-Un: no elastic shoulder band with unstable support, ESB-Un: elastic shoulder band with unstable support

exercise to be applied even to subjects with less muscle strength, as well as to individuals who require initial rehabilitation.

\section{Data analysis}

The data were analyzed by repeated one-way ANOVA, with a statistical significance of $p<.05$. SPSS 20.0 (version 20 for Windows; SPSS Inc., Chicago, IL, USA) was used for all statistical analysis.

\section{Results}

In order to compare stabilizing exercises with and without the ESB, the muscle activities of SA, UT, and LT were measured during the push-up plus exercise on stable and on unstable support. The ESB had no significant effect on activity of the UT or the LT on stable or unstable support $(p>.05)$. However, using the ESB on stable support resulted in a significant increase in SA activity compared to that on unstable support without $\mathrm{ESB}(p=.035)$ (Table 2).

\section{Discussion}

The results of our study showed that using the ESB while performing the push-up plus exercise on stable support induced no changes in UT and LT activities, but led to a significant increase in SA activity. We hypothesized that muscle activity would be higher when using the ESB on stable support than unstable support; however, the activity was significantly higher than that on unstable support without the band.

Similar to previous studies, our findings revealed no differences among SA activities on stable or unstable support. Lehman et al. (2008) examined subjects performing push-ups and push-up plus exercises and found no significant difference in SA activity on stable and unstable support. Sandhu et al. (2008) also reported the same result. In addition, no significant differences were observed in a study comparing SA activity for only the push-up plus exercise on stable and unstable support (Kim et al., 2014).

Conversely, a study by Park and Yoo (2011) revealed that SA activity increased significantly when the push-up plus exercise was performed on unstable support; however, this study differs from ours in that they examined the upper and lower SA separately and observed no significant difference in the more commonly studied upper SA, but observed a significant increase in the lower SA activity. In a study comparing sling and stable support, UT and SA activities increased simultaneously when performing the push-up plus exercise in a sling, but there was no selective beneficial effect on SA activity (Jeong et al., 2014). Increased UT activity causes clavicle retraction relative to the thorax, which can prevent scapulothoracic internal rotation. However, in healthy adults (Johnson et al., 1994), the UT only needs to contribute about $3^{\circ}$ during ST upward rotation at $50^{\circ}$ or more (Ludewig and Braman, 2011). Therefore, in patients with symptomatic pain, as 
seen in cases of insufficient ST upward rotation, UT strengthening exercises are not suitable. Conversely, SA has the largest momentum, generates scapular upward rotation torque (Johnson et al., 1994; Phadke et al., 2009), and weakness of the SA ultimately affects scapular posterior tilting (Ludewig and Braman, 2011).

For early rehabilitation in patients with symptomatic pain, it is not easy to directly apply the general phases of the push-up plus exercises (1. descending phase, 2. maintaining, 3. ascending phase). Nevertheless, many studies have used the above phases (Lehman et al., 2008; Park and Yoo, 2011). Thus, in the present study, we applied an ESB to the push-up plus exercise in the maintaining phase and measured UT, LT, and SA activities on different support types to test whether the ESB could be an effective instrument in rehabilitation.

When the ESB was applied to the push-up plus exercise on stable support, muscle activity increased significantly compared to the push-up plus exercise without the ESB on unstable support. These findings indicate that the ESB can provide sufficient resistance to increase SA activity.

Following shoulder injury, providing appropriate resistance during rehabilitation is very important, and applying a load of over $35 \%$ to the shoulder joint can delay rehabilitation (Ludewig et al., 2004). Tucker et al. (2010) claimed that, in patients with shoulder impingement (symptomatic subjects), changing the surface during closed kinetic exercises could have a negative effect on scapular muscle activities. Therefore, we believe that using the ESB on stable support rather than unstable support can be clinically applicable to increase SA activity.

It should be noted that our study had some limitations. Specifically, the sample size was small, and the intervention was applied to healthy subjects; therefore, we were unable to analyze the effects in patients with shoulder injury. These limitations will need to be overcome in future studies. In addition, it was difficult to objectively measure the elasticity of the ESB in each individual. Nevertheless, we sought to minimize this limitation by adjusting the band to a constant length relative to the subject's physical proportions. Because of the criterion of using healthy adult subjects, we excluded individuals with scapular winging. Thus, in future studies, it will be necessary to use the ESB in only individuals with scapular winging, and to examine not only activities of the shoulder stabilizing muscles, but also the UT/SA and LT/SA ratios.

\section{Conclusion}

We propose a study in which the ESB is applied to different phases of the exercise in other patients requiring shoulder stabilizing rehabilitation, as well as to the wall-press push-up plus exercises, in which there is no load from the weight of the body. In conclusion, the ESB designed in this study improved SA activity when applied to push-up plus exercises on stable support. Thus, it can potentially be used as an assistive tool for shoulder injury patients requiring initial rehabilitation. We believe that further research is needed in this area.

\section{References}

Bigliani LU, Codd TP, Connor PM, et al. Shoulder motion and laxity in the professional baseball player. Am J Sports Med. 1997;25(5):609-13.

Burkhart S, Morgan C, Kibler W. The disabled shoulder: spectrum of pathology part III: the SICK scapula, scapular dyskinesis, the kinetic chain, and rehabilitation. Arthroscopy. 2003;19(6): 641-61.

Cools AM, Declercq GA, Cambier DC, et al. Trapezius activity and intramuscular balance during isokinetic exercise in overhead athletes with impingement symptoms. Scand Med Sci Sports. 2007a;17(1):25-33.

Cools AM, Dewitte V, Lanszweert F, et al. Rehabilitation of scapular muscle balance: which exercise to prescribe? Am J Sports Med. 2007b;35(10):1744-51. 
Hsu YH, Chen WY, Lin HC, et al. The effects of taping on scapular kinematics and muscle performance in baseball players with shoulder impingement syndrome. J Electromyogr Kinesiol. 2009;19(6): 1092-9.

Jeong SY, Chung SH, Shim JH. Comparison of upper trapezius, anterior deltoid, and serratus anterior muscle activity during push-up plus exercises on slings and a stable surface. J Phys Ther Sci. 2014;26(6):937-9.

Johnson G, Bogduk N, Nowitzke A, et al. Anatomy and actions of the trapezius muscle. Clin Biomech. 1994; 9(1):44-50.

Kamkar A, Irrgang JJ, Whitney SL. Nonoperative management of secondary shoulder impingement syndrome. J Orthop Sports Phys Ther. 1993;17(5):212-24.

Kibler B. The role of the scapula in athletic shoulder function. Am J Sports Med. 1998;26(2):325-37.

Kim SH, Kwon OY, Kim SJ, et al. Serratus anterior muscle activation during knee push-up plus exercise performed on static stable, static unstable, and oscillating unstable surfaces in healthy subjects. Phys Ther Sport. 2014;15(1):20-5.

Lee SY, Lee DH, Park JS. The effect of hand position changes on electromyographic activity of shoulder stabilizers during push-up plus exercise on stable and unstable surfaces. J Physi Ther Sci. 2013;25(8):981-4.

Lehman GJ, Gilas D, Patel U. An unstable support surface does not increase scapulothoracic stabilizing muscle activity during push up and push up plus exercises. Man Ther. 2008;13(6):500-6.

Ludewig PM, Cook TM. Alterations in shoulder kinematics and associated muscle activity in people with symptoms of shoulder impingement. Phys Ther. 2000;80(3):276-91.

Ludewig PM, Hoff MS, Osowski EE, et al. Relative balance of serratus anterior and upper trapezius muscle activity during push-up exercises. Am J Sports Med. 2004; 32(2):484-93
Lugdewig PM, Braman JP. Shoulder impingement: biomechanical considerations in rehabilitation. Man Ther. 2011;16(1):33-9.

Moller CR, Huxel Bliven KC, Snyder Valier AR. Scapular muscle-activation ratios in patients with shoulder injuries during functional shoulder exercises. J Athl Train. 2014;49(3):345-55.

Paine R, Voight ML. The role of the scapula. Int J Sports Phys Ther. 2013;8(5):617-29.

Park SY, Yoo WG. Differential activation of parts of the serratus anterior muscle during push-up variations on stable and unstable bases of support. J Electromyogr Kinesiol. 2011;21(5):861-7.

Phadke V, Camargo PR, Ludewig PM. Scapular and rotator cuff function during arm elevation: a review of normal function and alterations with shoulder impingement. Rev Bras Fisioter. 2009;13(1):1-9.

Sandhu JS, Mahajan S, Shenoy S. An electromyographic analysis of shoulder muscle activation during push-up variations on stable and labile surfaces. Int J Shoulder Surg. 2008;2(2):30-5.

Seo SH, Jeon IH, Cho YH, et al. Surface EMG during the push-up plus exercise on a stable support or swiss ball: scapular stabilizer muscle exercise. J Phys Ther Sci. 2013;25(7):833-7.

Tucker WS, Armstrong CW, Cribble PA, et al. Scapular muscle activity in overhead athletes with symptoms of secondary shoulder impingement during closed chain exercises. Arch Phys Med Rehabil. 2010;91(4):550-6.

Voight ML, Thomson BC. The role of the scapula in the rehabilitation of shoulder injuries. J Athl Training. 2000;35(3):364-72.

Yoo IG, Yoo WG. The effect of a new neck support tying method using Thera-band on cervical ROM and shoulder muscle pain after overhead work. J Phys Ther Sci. 2013;25(7):843-4. 